Article

\title{
A Certain Mean Square Value Involving Dirichlet $L$-Functions
}

\author{
Wenpeng Zhang and Di Han *
}

School of Mathematics, Northwest University, Xi'an 710127, China; wpzhang@nwu.edu.cn

* Correspondence: dhan@nwu.edu.cn

Received: 7 May 2020; Accepted: 5 June 2020; Published: 9 June 2020

check for updates

\begin{abstract}
The main purpose of this article is using the elementary methods, the properties of Dirichlet $L$-functions to study the computational problem of a certain mean square value involving Dirichlet $L$-functions at positive integer points, and give some exact calculating formulae. As some applications, we obtain some interesting identities and inequalities involving character sums and trigonometric sums.
\end{abstract}

Keywords: Dirichlet $L$-functions; elementary method; mean square value; trigonometric sums; computational formula

\section{Introduction}

Let $q \geq 3$ be an integer, $\chi$ denotes a Dirichlet character $\bmod q$. Then Dirichlet $L$-functions $L(s, \chi)$ (see [1]) defined for $\operatorname{Re}(s)=\operatorname{Re}(\sigma+i t)=\sigma>1$ by the series

$$
L(s, \chi)=\sum_{n=1}^{\infty} \frac{\chi(n)}{n^{s}}
$$

where $s$ denotes a complex number, and $i^{2}=-1$.

If $\chi$ is any non-principal character mod $q$, then $L(s, \chi)$ is an entire function of $s$. If $\chi=\chi_{0}$ is the principal character mod $q$, then $L(s, \chi)$ is analytic everywhere except for a simple pole at $s=1$ with residue $\frac{\phi(q)}{q}, \phi(q)$ denotes the Euler function.

As we all know, Dirichlet $L$-functions play a very important role in the research of analytic number theory, many famous problems of number theory are closely related to it. For example, the famous Goldbach's conjecture, the distribution of twins prime and so on. Because of the importance of these functions, many scholars have studied its various properties, and obtained a series of important results. For example, M. Bordignon [2,3] studied the explicit bounds on exceptional zeroes of Dirichlet L-functions, and obtained a sharp upper bound estimate.

J. Andrade and S. Baluyot [4] proved some new results for the small zeros of Dirichlet $L$-functions of quadratic characters of prime modulus.

W. P. Zhang [5] proved that for any integer $q \geq 3$, one has the identity

$$
\sum_{\substack{\chi \bmod q \\ \chi(-1)=-1}}|L(1, \chi)|^{2}=\frac{\pi^{2}}{12} \frac{\phi^{2}(q)}{q^{2}}\left[q \cdot \prod_{p \mid q}\left(1+\frac{1}{p}\right)-3\right]
$$

where $\sum_{\substack{\chi \bmod q \\ \chi(-1)=-1}}$ denotes the summation over all odd characters $\bmod q$ (i.e., $\left.\chi(-1)=-1\right), \prod_{p \mid q}$ denotes the product over all different prime divisors of $q$. 
W. P. Zhang [6] introduced a generalized Dedekind sums, then he used the properties of this generalized Dedekind sums to prove the following identities

$$
\sum_{\substack{\chi \bmod q \\ \chi(-1)=1}}|L(2, \chi)|^{2}=\frac{\pi^{4}}{180} \cdot \phi(q) \prod_{p \mid q}\left(1-\frac{1}{p^{2}}\right)\left[\prod_{p \mid q}\left(1+\frac{1}{p^{2}}\right)+\frac{10}{q^{2}}\right]
$$

and

$$
\sum_{\substack{\chi \bmod q \\ \chi(-1)=-1}}|L(3, \chi)|^{2}=\frac{\pi^{6}}{1890} \cdot \phi(q) \prod_{p \mid q}\left(1-\frac{1}{p^{6}}\right)-\frac{\pi^{6}}{90} \cdot \frac{\phi(q)}{q^{4}} \prod_{p \mid q}\left(1-\frac{1}{p^{2}}\right),
$$

where $\sum_{\chi \bmod q}$ denotes the summation over all even characters mod $q$.

$\chi(-1)=1$

$X$. Lin [7] also proved a general mean square value formula for Dirichlet $L$-functions $L(n, \chi)$. That is, for any positive integer $k$, she obtained an exact calculating formula for

$$
\sum_{\substack{\chi \bmod q \\ \chi(-1)=1}}|L(2 k, \chi)|^{2} \text { and } \sum_{\substack{\chi \bmod q \\ \chi(-1)=-1}}|L(2 k-1, \chi)|^{2} .
$$

But the formulae in [7] are very long, and they do not look too beautiful. Especially, the calculation of coefficients in the formulae are very complicated. Therefore, in order to save the space, there is no need to list them here.

Some other papers related to $L$-functions can be found in references [7-16], we do not want to list them all here.

Very recently, W. P. Zhang and D. Han [17] studied the computational problems of the reciprocal sums of one kind of Chebyshev polynomials, and proved some interesting identities. Some of them are as follows:

Let $q$ be an odd number with $q \geq 3$. Then for any positive integer $k$ and integer $h$ with $(h, q)=1$, one has the identities

$$
\begin{aligned}
& \sum_{a=1}^{q-1} \frac{1}{\sin ^{2 k}\left(\frac{\pi h a}{q}\right)}=\sum_{a=1}^{q-1} \frac{1}{\sin ^{2 k}\left(\frac{\pi a}{q}\right)} \\
= & \frac{2}{(2 k-1) !} \sum_{u=1}^{k} \frac{S(k-1, k-u) \cdot(2 u-1) ! \cdot\left(q^{2 u}-1\right)}{\pi^{2 u}} \zeta(2 u)
\end{aligned}
$$

and

$$
\begin{aligned}
& \sum_{a=1}^{q-1} \frac{1}{\cos ^{2 k}\left(\frac{\pi h a}{q}\right)}=\sum_{a=1}^{q-1} \frac{1}{\cos ^{2 k}\left(\frac{\pi a}{q}\right)} \\
= & \frac{2}{(2 k-1) !} \cdot \sum_{u=1}^{k} \frac{S(k-1, k-u) \cdot\left(2^{2 u}-1\right) \cdot(2 u-1) ! \cdot\left(q^{2 u}-1\right)}{\pi^{2 u}} \cdot \zeta(2 u),
\end{aligned}
$$

where $\zeta(s)$ denotes the Riemann zeta-function, and the constants $S(k-1, u)$ are defined as $\prod_{u=0}^{k-1}\left(x+(2 u)^{2}\right)=$ $\sum_{u=0}^{k-1} S(k-1, u) \cdot x^{k-u}$, and $S(0,0)=1$.

The special cases in [17] has also been studied by Y. K. Ma and X. X. Lv [18]. That is, they studied the cases $k=1,2$ and 3 , and obtained some identities.

This paper, as a note of $[7,17]$, we will use the elementary methods and the properties of Dirichlet $L$-functions to study the computational problem of one kind of special mean square value of Dirichlet $L$-functions, and give a new and exact calculating formula for it. As some applications, we obtain two interesting inequalities involving character sums and trigonometric sums. That is, we will prove the following two conclusions: 
Theorem 1. Let $q>2$ be an integer and $\chi$ denote a Dirichlet character mod $q$. Then for any integer $k \geq 1$, we have the identity

$$
\begin{aligned}
& \sum_{\substack{\chi \bmod q \\
\chi(-1)=1}}\left|\sum_{u=1}^{k} \pi^{-2 u} \cdot \sigma_{k-1, k-u} \cdot(2 u-1) ! \cdot q^{2 u} \cdot L(2 u, \chi)\right|^{2} \\
& =\frac{\phi(q) \cdot((2 k-1) !)^{2}}{2 \cdot(4 k-1) !} \sum_{u=1}^{2 k} \pi^{-2 u} \cdot \sigma_{2 k-1,2 k-u} \cdot(2 u-1) ! \cdot q^{2 u} \cdot L\left(2 u, \chi_{0}\right),
\end{aligned}
$$

where $\chi_{0}$ denotes the principal character mod $q$, and the constants $\sigma_{k, u}$ are defined by

$$
\begin{aligned}
& \sigma_{k, 0}=1, \sigma_{k, 1}=\sum_{u=1}^{k}(2 u)^{2}, \sigma_{k, 2}=\sum_{1 \leq u<v \leq k}(2 u)^{2} \cdot(2 v)^{2}, \sigma_{k, 3}=\sum_{1 \leq u<v<w \leq k}(2 u)^{2} \cdot(2 v)^{2} \cdot(2 w)^{2}, \\
& \ldots \ldots, \sigma_{k, k}=\prod_{u=1}^{k}(2 u)^{2}=2^{2} \cdot 4^{2} \cdot 6^{2} \cdots(2 k-2)^{2} \cdot(2 k)^{2} .
\end{aligned}
$$

Theorem 2. Let $q$ be an integer with $q \geq 3$. Then for any integer $k \geq 1$, we have the identity

$$
\begin{aligned}
& \sum_{\substack{\chi \bmod q \\
\chi(-1)=-1}}\left|\sum_{u=1}^{k} \pi^{-2 u} \cdot \sigma_{k-1, k-u} \cdot(2 u) ! \cdot q^{2 u+1} \cdot L(2 u+1, \chi)\right|^{2} \\
= & \frac{\phi(q) \cdot((2 k) !)^{2}}{2 \cdot(4 k+1) !} \cdot \sum_{u=1}^{2 k+1} \pi^{2-2 u} \cdot \sigma_{2 k, 2 k+1-u} \cdot(2 u-1) ! \cdot q^{2 u} \cdot L\left(2 u, \chi_{0}\right) \\
- & \frac{\phi(q) \cdot((2 k) !)^{2}}{2 \cdot(4 k-1) !} \sum_{u=1}^{2 k} \pi^{2-2 u} \cdot \sigma_{2 k-1,2 k-u} \cdot(2 u-1) ! \cdot q^{2 u} \cdot L\left(2 u, \chi_{0}\right) .
\end{aligned}
$$

The main difference between our results and X. Lin [7] lies in the form of the mean square value of $L$-functions. Feature of our results is that they are simple in form and easy to calculate. Note that $\left\{\sigma_{k, u}\right\}(0 \leq u \leq k)$ is the same as $\{S(k, u)\}(0 \leq u \leq k)$ in [17]. It can be calculated by the recursive formula $\sigma_{k, u+1}=(2 k)^{2} \cdot \sigma_{k-1, u}+\sigma_{k-1, u+1}$ for all integers $0 \leq u \leq k-2, \sigma_{k, 0}=1$ and $\sigma_{k, k}=4^{k} \cdot(k !)^{2}$. To better understand the consequences of these theorems, we can use Mathematica software to calculate the value of $\sigma_{k, u}$ for all integers $0 \leq u \leq k$. Here we give partial values of $\sigma_{k, u}$ as shown in the following Table 1:

Table 1. Values of $\sigma_{k, u}$.

\begin{tabular}{cccccccc}
\hline$\sigma_{\boldsymbol{k}, \boldsymbol{u}}$ & $\boldsymbol{u}=\mathbf{0}$ & $\boldsymbol{u = 1}$ & $\boldsymbol{u = 2}$ & $\boldsymbol{u}=\mathbf{3}$ & $\boldsymbol{u}=\mathbf{4}$ & $\boldsymbol{u}=\mathbf{5}$ & $\boldsymbol{u}=\mathbf{6}$ \\
\hline$k=0$ & 1 & & & & & & \\
$k=1$ & 1 & 4 & & & & & \\
$k=2$ & 1 & 20 & 64 & & & & \\
$k=3$ & 1 & 56 & 784 & 2304 & & & \\
$k=4$ & 1 & 120 & 4368 & 52,480 & 147,456 & & \\
$k=5$ & 1 & 220 & 16,368 & 489,280 & $5,395,456$ & $14,745,600$ & \\
$k=6$ & 1 & 364 & 48,048 & $2,846,272$ & $75,851,776$ & $791,691,264$ & $2,123,366,400$ \\
\hline
\end{tabular}

If $\chi_{0}$ is the principal character modulo $q$ with $q>1$, then note the identities

$$
L\left(2 k, \chi_{0}\right)=\zeta(2 k) \cdot \prod_{p \mid q}\left(1-\frac{1}{p^{2 k}}\right)
$$

and

$$
\zeta(2 k)=(-1)^{k+1} \cdot \frac{(2 \pi)^{2 k} \cdot B_{2 k}}{2 \cdot(2 k) !} \text { for all positive integers } k,
$$

where $B_{2 k}$ denotes the Bernoulli numbers, and $\prod_{p \mid q}$ denotes the product over all distinct prime divisors of $q$. Therefore, for any fixed positive integer $k$, we can give the exact values in Theorems 1 and 2 . Especially for integers $k=1$ and 2 , from these theorems we may immediately deduce the following results: 
Corollary $1([6,7])$. Let $q$ be an integer with $q \geq 3$, then we have the identity

$$
\sum_{\substack{\chi \bmod q \\ \chi(-1)=1}}|L(2, \chi)|^{2}=\frac{\pi^{2}}{3} \cdot \frac{\phi(q)}{q^{2}} \cdot L\left(2, \chi_{0}\right)+\frac{\phi(q)}{2} \cdot L\left(4, \chi_{0}\right) .
$$

Corollary 2. Let $q$ be an integer with $q \geq 3$, then we have the identity

$$
\begin{aligned}
& \sum_{\substack{\chi \bmod q \\
\chi(-1)=1}}\left|2 L(2, \chi)+\frac{3 q^{2}}{\pi^{2}} \cdot L(4, \chi)\right|^{2}=\frac{72 \pi^{2}}{35} \cdot \frac{\phi(q)}{q^{2}} \cdot L\left(2, \chi_{0}\right) \\
& \quad+\frac{21}{5} \cdot \phi(q) \cdot L\left(4, \chi_{0}\right)+\frac{6}{\pi^{2}} \cdot \phi(q) \cdot q^{2} \cdot L\left(6, \chi_{0}\right)+\frac{9}{2 \cdot \pi^{4}} \cdot \phi(q) \cdot q^{4} \cdot L\left(8, \chi_{0}\right) .
\end{aligned}
$$

Corollary 3 ([6]). Let $q$ be an integer with $q \geq 3$, then we have the identity

$$
\sum_{\substack{\chi \bmod q \\ \chi(-1)=-1}}|L(1, \chi)|^{2}=\frac{\phi(q)}{2} L\left(2, \chi_{0}\right)-\frac{\pi^{2}}{4} \frac{\phi^{2}(q)}{q^{2}}=\frac{\pi^{2}}{12} \frac{\phi^{2}(q)}{q^{2}}\left[q \prod_{p \mid q}\left(1+\frac{1}{p}\right)-3\right] .
$$

Of course, Corollary 3 is not a corollary of Theorem 1 and 2, but rather a corollary of their proofs (and the lemmas building up to the proofs).

Corollary $4([6,7])$. Let $q$ be an integer with $q \geq 3$, then we have the identity

$$
\sum_{\substack{\chi \bmod q \\ \chi(-1)=-1}}|L(3, \chi)|^{2}=\frac{1}{2} \cdot \phi(q) \cdot L\left(6, \chi_{0}\right)-\frac{\pi^{4}}{15} \cdot \frac{\phi(q)}{q^{4}} \cdot L\left(2, \chi_{0}\right) .
$$

Corollary 5. Let $q$ be an integer with $q \geq 3$, then for any integer $h$ with $(h, q)=1$ and $\chi$ mod $q$, we have the estimate

$$
\left|\sum_{a=1}^{q-1} \frac{\chi(a)}{\sin ^{2}\left(\frac{\pi h a}{q}\right)}\right| \leq \frac{2 \cdot q^{2}}{\pi^{2}} \cdot|L(2, \chi)| \leq \frac{1}{3} \cdot q^{2} \cdot \prod_{p \mid q}\left(1-\frac{1}{p^{2}}\right) .
$$

Corollary 6. Let $q$ be an integer with $q \geq 3$, then for any integer $h$ with $(h, q)=1$ and $\chi$ mod $q$, we have the estimate

$$
\left|\sum_{a=1}^{q-1} \frac{\chi(a)}{\sin ^{4}\left(\frac{\pi h a}{q}\right)}\right| \leq \frac{2 \cdot q^{4}}{\pi^{4}} \cdot|L(4, \chi)|+\frac{4 \cdot q^{2}}{3 \cdot \pi^{2}} \cdot|L(2, \chi)| .
$$

Especially, if $p$ is an odd prime, then we have the estimate

$$
\left|\sum_{a=1}^{p-1} \frac{\chi(a)}{\sin ^{4}\left(\frac{\pi h a}{p}\right)}\right| \leq \frac{\left(p^{2}-1\right)\left(p^{2}+11\right)}{45} .
$$

Corollary 7. Let $\chi_{2}$ denotes the Legendre's symbol mod 5. Then we have

$$
L\left(2, \chi_{2}\right)=\frac{4 \cdot \pi^{2}}{25 \cdot \sqrt{5}} .
$$

Corollary 8. Let $\chi_{2}$ denotes the Legendre's symbol mod 3. Then we have

$$
L\left(1, \chi_{2}\right)=\frac{\pi}{3 \cdot \sqrt{3}} \text { and } L\left(3, \chi_{2}\right)=\frac{4 \cdot \pi^{3}}{81 \cdot \sqrt{3}} \text {. }
$$




\section{Several Lemmas}

In this section, we shall give several simple lemmas, they are used in the proofs of our theorems. First we have the following:

Lemma 1. Let $q>3$ be an integer, $\chi$ be any non-principal character mod $q$, and $f(s)=\ln \frac{1}{\sin (\pi s)}$. Then for any positive integer $k$, we have the identities

$$
\sum_{a=1}^{q-1} \chi(a) \cdot f^{(2 k-1)}\left(\frac{a}{q}\right)= \begin{cases}-2(2 k-2) ! \cdot q^{2 k-1} \cdot L(2 k-1, \chi), & \text { if } \chi(-1)=-1 \\ 0, & \text { if } \chi(-1)=1\end{cases}
$$

and

$$
\sum_{a=1}^{q-1} \chi(a) \cdot f^{(2 k)}\left(\frac{a}{q}\right)= \begin{cases}2(2 k-1) ! \cdot q^{2 k} \cdot L(2 k, \chi), & \text { if } \chi(-1)=1 \\ 0, & \text { if } \chi(-1)=-1\end{cases}
$$

where $f^{(k)}(s)$ denotes the $k$-order derivative of $f(s)$, and $L(n, \chi)$ denotes the Dirichlet L-function corresponding to $\chi$ mod $q$.

Proof of Lemma 1. From the definition and properties of the derivative of $f(s)$ we have

$$
f^{\prime}(s)=-\pi \cdot \frac{\cos (\pi s)}{\sin (\pi s)}=-\pi \cdot \cot (\pi s) \text { and } f^{\prime \prime}(s)=\frac{\pi^{2}}{\sin ^{2}(\pi s)}, 0<s<1 .
$$

On the other hand, we have (see Corollary 6, Section 3, Chapter 5 in [12])

$$
\sin (\pi s)=\pi s \cdot \prod_{n=1}^{\infty}\left(1-\frac{s^{2}}{n^{2}}\right)
$$

and it is convergent for all $0<s<1$.

From (2) and the properties of the derivative we also have

$$
f^{\prime}(s)=-\frac{1}{s}-\sum_{n=1}^{\infty}\left(\frac{1}{n+s}-\frac{1}{n-s}\right)
$$

and

$$
f^{\prime \prime}(s)=\frac{1}{s^{2}}+\sum_{n=1}^{\infty}\left(\frac{1}{(n+s)^{2}}+\frac{1}{(n-s)^{2}}\right) .
$$

In general, for any positive integer $k$, we have

$$
f^{(2 k-1)}(s)=-(2 k-2) ! \cdot\left(\frac{1}{s^{2 k-1}}+\sum_{n=1}^{\infty}\left(\frac{1}{(n+s)^{2 k-1}}-\frac{1}{(n-s)^{2 k-1}}\right)\right)
$$

and

$$
f^{(2 k)}(s)=(2 k-1) ! \cdot\left(\frac{1}{s^{2 k}}+\sum_{n=1}^{\infty}\left(\frac{1}{(n+s)^{2 k}}+\frac{1}{(n-s)^{2 k}}\right)\right) .
$$

Taking $s=\frac{a}{q}$, if $\chi$ is an odd character $\bmod q$, note that $\chi(q-1)=\chi(-1)=-1$, then from (5) and the definition of Dirichlet $L$-function we have

$$
\begin{aligned}
& \sum_{a=1}^{q-1} \chi(a) \cdot f^{(2 k-1)}\left(\frac{a}{q}\right) \\
= & -(2 k-2) ! \cdot \sum_{a=1}^{q-1} \chi(a) \cdot\left(\frac{q^{2 k-1}}{a^{2 k-1}}+\sum_{n=1}^{\infty}\left(\frac{1}{\left(n+\frac{a}{q}\right)^{2 k-1}}-\frac{1}{\left(n-\frac{a}{q}\right)^{2 k-1}}\right)\right) \\
= & -(2 k-2) ! \cdot q^{2 k-1} \cdot \sum_{a=1}^{q-1} \chi(a) \cdot\left(\sum_{n=0}^{\infty} \frac{1}{(q n+a)^{2 k-1}}-\sum_{n=1}^{\infty} \frac{1}{(n q-a)^{2 k-1}}\right)
\end{aligned}
$$




$$
\begin{aligned}
& =-(2 k-2) ! \cdot q^{2 k-1} \cdot\left(\sum_{n=0}^{\infty} \sum_{a=0}^{q-1} \frac{\chi(a)}{(n q+a)^{2 k-1}}+\sum_{n=0}^{\infty} \sum_{a=0}^{q-1} \frac{\chi(q-a)}{(n q+q-a)^{2 k-1}}\right) \\
& =-2(2 k-2) ! \cdot q^{2 k-1} \cdot \sum_{n=1}^{\infty} \frac{\chi(n)}{n^{2 k-1}} \\
& =-2(2 k-2) ! \cdot q^{2 k-1} \cdot L(2 k-1, \chi), k \geq 2 .
\end{aligned}
$$

If $k=1$, then from the relationship between the Dirichlet $L$-function $L(s, \chi)$ and the Hurwitz zeta-function $\zeta(s, \alpha)$ we can also deduce (7) is correct.

It is clear that $f^{\prime \prime}(s)$ is an even function, and for any integer $n, f^{\prime \prime}(n+s)=f^{\prime \prime}(s)$. So for $k \geq 2, f^{(2 k-1)}(s)$ must be an odd function. If $\chi(-1)=1$, then we have

$$
\begin{aligned}
& \sum_{a=1}^{q-1} \chi(a) \cdot f^{(2 k-1)}\left(\frac{a}{q}\right)=\sum_{a=1}^{q-1} \chi(-a) \cdot f^{(2 k-1)}\left(\frac{q-a}{q}\right) \\
= & -\sum_{a=1}^{q-1} \chi(a) \cdot f^{(2 k-1)}\left(\frac{a}{q}\right)=0 .
\end{aligned}
$$

This identity is also correct, if $k=1$.

Similarly, if $\chi$ is an even character $\bmod q$, note that $\chi(q-1)=\chi(-1)=1$, then from (6) and the definition of Dirichlet $L$-function we have

$$
\sum_{a=1}^{q-1} \chi(a) \cdot f^{(2 k)}\left(\frac{a}{q}\right)=2(2 k-1) ! \cdot q^{2 k} \cdot L(2 k, \chi) .
$$

If $\chi$ is an odd character mod $q$, then $\chi(-1)=-1$ and

$$
\sum_{a=1}^{q-1} \chi(a) \cdot f^{(2 k)}\left(\frac{a}{q}\right)=0
$$

Now Lemma 1 follows from (7)-(10).

Lemma 2. Let $f(s)=\ln \frac{1}{\sin (\pi s)}$. Then for any positive integer $k$, we have the identities

$$
\begin{gathered}
\text { (A). } \quad \sum_{u=0}^{k} \pi^{2 u} \cdot \sigma_{k, u} \cdot f^{(2 k+2-2 u)}(s)=\frac{(2 k+1) ! \cdot \pi^{2 k+2}}{\sin ^{2 k+2}(\pi s)} \\
\text { (B). } \quad \sum_{u=0}^{k-1} \pi^{2 u} \cdot \sigma_{k-1, u} \cdot f^{(2 k-2 u+1)}(s)=-\frac{(2 k) ! \cdot \pi^{2 k+1} \cdot \cos (\pi s)}{\sin ^{2 k+1}(\pi s)}
\end{gathered}
$$

where $\sigma_{k, 0}=1, \sigma_{k, 1}=\sum_{u=1}^{k}(2 u)^{2}, \sigma_{k, 2}=\sum_{1 \leq u<v \leq k}(2 u)^{2} \cdot(2 v)^{2}, \sigma_{k, 3}=\sum_{1 \leq u<v<w \leq k}(2 u)^{2} \cdot(2 v)^{2} \cdot(2 w)^{2}, \cdots, \sigma_{k, k}=$ $\prod_{u=1}^{k}(2 u)^{2}=2^{2} \cdot 4^{2} \cdot 6^{2} \cdots(2 k-2)^{2} \cdot(2 k)^{2}$.

Proof of Lemma 2. First we prove (A) in Lemma 2 by mathematical induction. From (1) we have

$$
f^{\prime \prime}(s)=\frac{\pi^{2}}{\sin ^{2}(\pi s)} \text { and } f^{(4)}(s)=\frac{6 \cdot \pi^{4}}{\sin ^{4}(\pi s)}-\frac{4 \cdot \pi^{4}}{\sin ^{2}(\pi s)} .
$$

That is,

$$
f^{(4)}(s)+2^{2} \cdot \pi^{2} \cdot f^{\prime \prime}(s)=\frac{3 ! \cdot \pi^{4}}{\sin ^{4}(\pi s)}
$$

So Lemma 2 is correct for $k=1$. Assume Lemma 2 is correct for $k \geq 2$. That is,

$$
\sum_{u=0}^{k} \pi^{2 u} \cdot \sigma_{k, u} \cdot f^{(2 k-2 u+2)}(s)=\frac{(2 k+1) ! \cdot \pi^{2 k+2}}{\sin ^{2 k+2}(\pi s)} .
$$


Then from (12) and the definition of the derivative we have

$$
\sum_{u=0}^{k} \pi^{2 u} \cdot \sigma_{k, u} \cdot f^{(2 k-2 u+3)}(s)=-(2 k+2) ! \cdot \pi^{2 k+3} \frac{\cos (\pi s)}{\sin ^{2 k+3}(\pi s)} .
$$

Note that $\cos ^{2}(\pi s)+\sin ^{2}(\pi s)=1$. Hence, from (13) we have

$$
\begin{aligned}
& \sum_{u=0}^{k} \pi^{2 u} \cdot \sigma_{k, u} \cdot f^{(2 k-2 u+4)}(s)=\frac{(2 k+2) ! \cdot \pi^{2 k+4}}{\sin ^{2 k+2}(\pi s)}+\frac{(2 k+3) ! \cdot \pi^{2 k+4} \cdot \cos ^{2}(\pi s)}{\sin ^{2 k+4}(\pi s)} \\
& =\frac{(2 k+3) ! \cdot \pi^{2 k+4}}{\sin ^{2 k+4}(\pi s)}-\frac{(2 k+2)^{2} \cdot(2 k+1) ! \cdot \pi^{2 k+4}}{\sin ^{2 k+2}(\pi s)} .
\end{aligned}
$$

Combining (12) and (14) we have

$$
\begin{aligned}
& \sum_{u=0}^{k} \pi^{2 u} \cdot \sigma_{k, u} \cdot f^{(2 k-2 u+4)}(s)+(2 k+2)^{2} \cdot \pi^{2} \cdot\left(\sum_{u=0}^{k} \pi^{2 u} \cdot \sigma_{k, u} \cdot f^{(2 k-2 u+2)}(s)\right) \\
& =\sum_{u=0}^{k-1} \pi^{2 u+2} \cdot \sigma_{k, u+1} \cdot f^{(2 k-2 u+2)}(s)+\sum_{u=0}^{k-1} \pi^{2 u+2} \cdot(2 k+2)^{2} \cdot \sigma_{k, u} \cdot f^{(2 k-2 u+2)}(s) \\
& +f^{(2 k+4)}(s)+(2 k+2)^{2} \cdot \pi^{2 k+2} \cdot \sigma_{k, k} \cdot f^{\prime \prime}(s) \\
& =\sum_{u=0}^{k+1} \pi^{2 u} \cdot \sigma_{k+1, u} \cdot f^{(2 k-2 u+4)}(s)=\frac{(2 k+3) ! \cdot \pi^{2 k+4}}{\sin ^{2 k+4}(\pi s)},
\end{aligned}
$$

where we have used the identity $\sigma_{k+1,0}=\sigma_{k, 0}=1, \sigma_{k+1, k+1}=(2 k+2)^{2} \cdot \sigma_{k, k}$ and $\sigma_{k+1, u+1}=\sigma_{k, u+1}+(2 k+2)^{2}$. $\sigma_{k, u}$ for all positive integers $0 \leq u \leq k-1$. It is clear this formula implies that Lemma 2 is correct for positive $k+1$. This proves (A) in Lemma 2 by mathematical induction.

To prove (B), we substitute $k+1$ by $k$ in (A), and then taking the derivative of (A), and that gives us (B). This proves Lemma 2.

Lemma 3. Let $q>1$ be an integer and $\chi$ be any even character mod $q$. Then for any integer $k \geq 0$, we have the identity

$$
\begin{aligned}
& 2 \sum_{u=0}^{k} \pi^{2 u} \cdot \sigma_{k, u} \cdot(2 k+2-2 u-1) ! \cdot q^{2(k+1-u)} \cdot L(2 k+2-2 u, \chi) \\
& =(2 k+1) ! \cdot \pi^{2 k+2} \cdot \sum_{a=1}^{q-1} \frac{\chi(a)}{\sin ^{2 k+2}\left(\frac{\pi a}{q}\right)}
\end{aligned}
$$

If $\chi$ is any odd Dirichlet character mod $q$ (i.e., $\chi(-1)=-1$ ), then we have

$$
\begin{aligned}
& 2 \sum_{u=1}^{k} \pi^{2(k-u)} \cdot \sigma_{k-1, k-u} \cdot(2 u) ! \cdot q^{2 u+1} \cdot L(2 u+1, \chi) \\
& =(2 k) ! \cdot \pi^{2 k+1} \sum_{a=1}^{q-1} \chi(a) \cdot \frac{\cos \left(\frac{\pi a}{q}\right)}{\sin ^{2 k+1}\left(\frac{\pi a}{q}\right)}
\end{aligned}
$$

where $L(n, \chi)$ denotes the Dirichlet L-function corresponding to $\chi$ mod $q$.

Proof of Lemma 3. Here we only treat the case $k \geq 1$. The case $k=0$ is dealt with separately. First if $\chi(-1)=1$, then from Lemma 1 and (A) of Lemma 2 we have

$$
\sum_{u=0}^{k} \pi^{2 u} \cdot \sigma_{k, u} \cdot \sum_{a=1}^{q-1} \chi(a) f^{(2 k+2-2 u)}\left(\frac{a}{q}\right)=(2 k+1) ! \cdot \pi^{2 k+2} \cdot \sum_{a=1}^{q-1} \frac{\chi(a)}{\sin ^{2 k+2}\left(\frac{\pi a}{q}\right)}
$$

or the identity 


$$
\begin{aligned}
& 2 \sum_{u=0}^{k} \pi^{2 u} \cdot \sigma_{k, u} \cdot(2 k-2 u+1) ! \cdot q^{2(k+1-u)} \cdot L(2 k+2-2 u, \chi) \\
& =(2 k+1) ! \cdot \pi^{2 k+2} \cdot \sum_{a=1}^{q-1} \frac{\chi(a)}{\sin ^{2 k+2}\left(\frac{\pi a}{q}\right)}
\end{aligned}
$$

This proves the first formula in Lemma 3.

If $\chi$ is an odd character mod $q$, then from Lemma 1 and (B) of Lemma 2 we also have the identity

$$
\begin{aligned}
& \sum_{u=0}^{k-1} \pi^{2 u} \cdot \sigma_{k-1, u} \cdot \sum_{a=1}^{q-1} \chi(a) \cdot f^{(2 k-2 u+1)}\left(\frac{a}{q}\right) \\
& =-2 \sum_{u=0}^{k-1} \pi^{2 u} \cdot \sigma_{k-1, u} \cdot(2 k-2 u) ! \cdot q^{2 k-2 u+1} \cdot L(2 k-2 u+1, \chi) \\
& =-(2 k) ! \cdot \pi^{2 k+1} \sum_{a=1}^{q-1} \chi(a) \cdot \frac{\cos \left(\frac{\pi a}{q}\right)}{\sin ^{2 k+1}\left(\frac{\pi a}{q}\right)} .
\end{aligned}
$$

That is, we have the identity

$$
\begin{aligned}
& 2 \sum_{u=1}^{k} \pi^{2(k-u)} \cdot \sigma_{k-1, k-u} \cdot(2 u) ! \cdot q^{2 u+1} \cdot L(2 u+1, \chi) \\
& =(2 k) ! \cdot \pi^{2 k+1} \sum_{a=1}^{q-1} \chi(a) \cdot \frac{\cos \left(\frac{\pi a}{q}\right)}{\sin ^{2 k+1}\left(\frac{\pi a}{q}\right)} .
\end{aligned}
$$

If $k=0$, then for $\chi(-1)=-1$, from [5] (Lemma 2) we have

$$
L(1, \chi)=\frac{\pi}{2 q} \sum_{a=1}^{q} \chi(a) \cdot \cot \left(\frac{\pi a}{q}\right)=\frac{\pi}{2 q} \sum_{a=1}^{q} \chi(a) \cdot \frac{\cos \left(\frac{\pi a}{q}\right)}{\sin \left(\frac{\pi a}{q}\right)} .
$$

This means that Lemma 3 is also correct.

This completes the proof of Lemma 3.

\section{Proofs of the Theorems}

In this section, we shall complete the proofs of our theorems. First if $\chi(-1)=1$, then from the first formula in Lemma 3 we have

$$
\begin{aligned}
& 2 \sum_{u=1}^{k} \pi^{2(k-u)} \cdot \sigma_{k-1, k-u} \cdot(2 u-1) ! \cdot q^{2 u} \cdot L(2 u, \chi) \\
& =(2 k-1) ! \cdot \pi^{2 k} \cdot \sum_{a=1}^{q-1} \frac{\chi(a)}{\sin ^{2 k}\left(\frac{\pi a}{q}\right)}
\end{aligned}
$$

and

$$
\begin{aligned}
& 2 \sum_{u=1}^{2 k} \pi^{2(2 k-u)} \cdot \sigma_{2 k-1,2 k-u} \cdot(2 u-1) ! \cdot q^{2 u} \cdot L\left(2 u, \chi_{0}\right) \\
& =(4 k-1) ! \cdot \pi^{4 k} \cdot \sum_{a=1}^{q-1} \frac{\chi_{0}(a)}{\sin ^{4 k}\left(\frac{\pi a}{q}\right)},
\end{aligned}
$$

where $\chi_{0}$ denotes the principal character $\bmod q$.

Note that if $\chi(-1)=-1$, then we have the identity

$$
\sum_{a=1}^{q-1} \frac{\chi(a)}{\sin ^{2 k}\left(\frac{\pi a}{q}\right)}=\sum_{a=1}^{q-1} \frac{\chi(q-a)}{\sin ^{2 k}\left(\frac{\pi(q-a)}{q}\right)}=-\sum_{a=1}^{q-1} \frac{\chi(a)}{\sin ^{2 k}\left(\frac{\pi a}{q}\right)}=0 .
$$


So from (15), (16) and the orthogonality of the characters we have

$$
\begin{aligned}
& \sum_{\substack{\chi \bmod q \\
\chi(-1)=1}}\left|\sum_{u=1}^{k} \pi^{2(k-u)} \cdot \sigma_{k-1, k-u} \cdot(2 u-1) ! \cdot q^{2 u} \cdot L(2 u, \chi)\right|^{2} \\
= & \frac{\left((2 k-1) ! \cdot \pi^{2 k}\right)^{2}}{4} \sum_{\chi \bmod q}\left|\sum_{a=1}^{q-1} \frac{\chi(a)}{\sin ^{2 k}\left(\frac{\pi a}{q}\right)}\right|^{2} \\
= & \frac{\phi(q) \cdot((2 k-1) !)^{2} \cdot \pi^{4 k}}{4} \cdot \sum_{a=1}^{q-1} \frac{\chi_{0}(a)}{\sin ^{4 k}\left(\frac{\pi a}{q}\right)} \\
= & \frac{\phi(q) \cdot((2 k-1) !)^{2}}{2 \cdot(4 k-1) !} \sum_{u=1}^{2 k} \pi^{2(2 k-u)} \cdot \sigma_{2 k-1,2 k-u} \cdot(2 u-1) ! \cdot q^{2 u} \cdot L\left(2 u, \chi_{0}\right) .
\end{aligned}
$$

This proves Theorem 1 .

Now we prove Theorem 2. If $\chi$ be any odd Dirichlet character mod $q$, then note the identity

$$
\sum_{\substack{\chi \bmod q \\ \chi(-1)=-1}}\left|\sum_{a=1}^{q-1} \chi(a) \cdot \frac{\cos \left(\frac{\pi a}{q}\right)}{\sin ^{2 k+1}\left(\frac{\pi a}{q}\right)}\right|^{2}=\sum_{\chi \bmod q}\left|\sum_{a=1}^{q-1} \chi(a) \cdot \frac{\cos \left(\frac{\pi a}{q}\right)}{\sin ^{2 k+1}\left(\frac{\pi a}{q}\right)}\right|^{2},
$$

where we have used the fact that the inner sum is 0 , if $\chi(-1)=1$.

From this identity, (15), the orthogonality of characters $\bmod q$ and the second formula of Lemma 3 we have

$$
\begin{aligned}
& 4 \sum_{\substack{\chi \bmod q \\
\chi(-1)=-1}}\left|\sum_{u=1}^{k} \pi^{2(k-u)} \cdot \sigma_{k-1, k-u} \cdot(2 u) ! \cdot q^{2 u+1} \cdot L(2 u+1, \chi)\right|^{2} \\
= & \left((2 k) ! \cdot \pi^{2 k+1}\right)^{2} \sum_{\chi \bmod q}\left|\sum_{a=1}^{q-1} \chi(a) \cdot \frac{\cos \left(\frac{\pi a}{q}\right)}{\sin ^{2 k+1}\left(\frac{\pi a}{q}\right)}\right|^{2} \\
= & \phi(q) \cdot((2 k) !)^{2} \cdot \pi^{4 k+2} \cdot \sum_{a=1}^{q-1} \frac{\chi_{0}(a) \cdot \cos ^{2}\left(\frac{\pi a}{q}\right)}{\sin ^{4 k+2}\left(\frac{\pi a}{q}\right)} \\
= & \phi(q) \cdot((2 k) !)^{2} \cdot \pi^{4 k+2} \cdot\left[\sum_{a=1}^{q-1} \frac{\chi_{0}(a)}{\sin ^{4 k+2}\left(\frac{\pi a}{q}\right)}-\sum_{a=1}^{q-1} \frac{\chi_{0}(a)}{\sin ^{4 k}\left(\frac{\pi a}{q}\right)}\right] \\
= & \frac{2 \cdot \phi(q) \cdot((2 k) !)^{2}}{(4 k+1) !} \cdot \sum_{u=1}^{2 k+1} \pi^{4 k+2-2 u} \cdot \sigma_{2 k, 2 k+1-u} \cdot(2 u-1) ! \cdot q^{2 u} \cdot L\left(2 u, \chi_{0}\right) \\
- & \frac{2 \pi^{2} \cdot \phi(q) \cdot((2 k) !)^{2}}{(4 k-1) !} \sum_{u=1}^{2 k} \pi^{2(2 k-u)} \cdot \sigma_{2 k-1,2 k-u} \cdot(2 u-1) ! \cdot q^{2 u} \cdot L\left(2 u, \chi_{0}\right) .
\end{aligned}
$$

This completes the proof of Theorem 2.

Now we prove Corollary 3. From (1) and Lemma 1 we have

$$
\sum_{a=1}^{q-1} \chi(a) f^{\prime}\left(\frac{a}{q}\right)=-\pi \cdot \sum_{a=1}^{q-1} \chi(a) \frac{\cos \left(\frac{\pi a}{q}\right)}{\sin \left(\frac{\pi a}{q}\right)}=-2 q \cdot L(1, \chi) .
$$

So from (17) and the orthogonality of the characters mod $q$ we have 


$$
\begin{aligned}
& 4 q^{2} \cdot \sum_{\substack{\chi \bmod q \\
\chi(-1)=-1}}|L(1, \chi)|^{2}=\sum_{\substack{\chi(-1)=-1 \\
\chi(-1)}}\left|-\pi \cdot \sum_{a=1}^{q-1} \chi(a) \frac{\cos \left(\frac{\pi a}{q}\right)}{\sin \left(\frac{\pi a}{q}\right)}\right|^{2} \\
& =\pi^{2} \cdot \phi(q) \cdot \sum_{a=1}^{q-1} \chi_{0}(a) \frac{\cos ^{2}\left(\frac{\pi a}{q}\right)}{\sin ^{2}\left(\frac{\pi a}{q}\right)}=\pi^{2} \cdot \phi(q) \cdot\left(\sum_{a=1}^{q-1} \frac{\chi_{0}(a)}{\sin ^{2}\left(\frac{\pi a}{q}\right)}-\phi(q)\right) \\
& =2 \cdot \phi(q) \cdot q^{2} \cdot L\left(2, \chi_{0}\right)-\pi^{2} \cdot \phi^{2}(q) .
\end{aligned}
$$

It is clear that (18) implies the identity

$$
\sum_{\substack{\chi \bmod q \\ \chi(-1)=-1}}|L(1, \chi)|^{2}=\frac{\phi(q)}{2} \cdot L\left(2, \chi_{0}\right)-\frac{\pi^{2}}{4} \cdot \frac{\phi^{2}(q)}{q^{2}} .
$$

Corollarys 5 and 6 can be deduced from Lemmas 1 and 2 . In fact if $\chi(-1)=1$, then from Lemma 1 with $k=1$ we have

$$
2 q^{2}|L(2, \chi)|=\left|\sum_{a=1}^{q-1} \chi(a) \cdot f^{\prime \prime}\left(\frac{a}{q}\right)\right|=\pi^{2}\left|\sum_{a=1}^{q-1} \frac{\chi(a)}{\sin ^{2}\left(\frac{\pi a}{q}\right)}\right|
$$

or

$$
\left|\sum_{a=1}^{q-1} \frac{\chi(a)}{\sin ^{2}\left(\frac{\pi a}{q}\right)}\right|=\frac{2 \cdot q^{2}}{\pi^{2}} \cdot|L(2, \chi)| \leq \frac{1}{3} \cdot q^{2} \cdot \prod_{p \mid q}\left(1-\frac{1}{p^{2}}\right)
$$

If $\chi(-1)=-1$, then we have

$$
\left|\sum_{a=1}^{q-1} \frac{\chi(a)}{\sin ^{2}\left(\frac{\pi a}{q}\right)}\right|=0
$$

Now Corollary 5 follows from (19) and (20).

Similarly, we can also deduce Corollary 6.

Corollarys 7 and 8 are the special cases of Theorems 1 and 2. In fact, for any odd prime $p$, it is clear that $\chi_{2}$ is a real character $\bmod p$, so $L\left(n, \chi_{2}\right)>0$ for all positive integers $n$. Of course, from [1] (Theorem 6.20) we can also get $L\left(1, \chi_{2}\right)>0$. If we taking $q=5$, then note that $\chi_{0}$ and $\chi_{2}$ are all even characters mod 5 , so from Theorem 1 we can easily deduce Corollary 7.

Corollary 8 follows from Theorem 2 with $q=3$.

This completes the proofs of our all results.

\section{Conclusions}

The main results of this article are two theorems and eight corollaries. Theorem 1 establishes a new hybrid mean square value formula involving Dirichlet $L$-function at the even point $s=2 k$. Theorem 2 establishes a new hybrid mean square value formula involving Dirichlet $L$-function at the odd point $s=2 k-1$. As some special cases or applications of these theorems, we give eight corollaries, some of these are existing results and some are new. In particular, Corollarys 5 and 6 give two strong upper bound estimates for a class of character sum, Corollarys 7 and 8 give two special values of Dirichlet $L$-functions at points $s=2$ and $s=3$. These are all new contributions to Dirichlet $L$-functions.

Author Contributions: W.Z. and D.H. have equally contributed to this work. All authors have read and agreed to the published version of the manuscript.

Funding: This work is supported by the N. S. F. (11771351, 11701447) and the Natural Science Basic Research Project in Shaanxi Province ( 2017JK1002) of P. R. China. 
Acknowledgments: The authors would like to thank the referee for their very helpful and detailed comments. In particular, many English grammar and error correction, so that the text reads more smoothly.

Conflicts of Interest: The authors declare that there are no conflicts of interest regarding the publication of this paper.

\section{References}

1. Apostol, T.M. Introduction to Analytic Number Theory; Springer: New York, NY, USA, 1976.

2. Bordignon, M. Explicit bounds on exceptional zeroes of Dirichlet L-functions. J. Number Theory 2019, 201, 68-76. [CrossRef]

3. Bordignon, M. Explicit bounds on exceptional zeroes of Dirichlet L-functions II. J. Number Theory 2019, 210, 481-487. [CrossRef]

4. Andrade, J.; Baluyot, S. Small zeros of Dirichlet L-functions of quadratic characters of prime modulus. Res. Number Theory 2020, 6, 18. [CrossRef]

5. Zhang, W.P. Lecture Notes in Contemporary Mathematics; Science Press: Beijing, China, 1989; pp. $173-179$.

6. Zhang, W.P. On the general Dedekind sums and one kind identities of Dirichlet L-functions. Acta Math. Sin. 2001, 44, 269-272. (In Chinese)

7. Lin, X. On the mean square value of the Dirichlet L-function at positive integers. Acta Arith. 2019, 189, 367-379. [CrossRef]

8. Walum, H. An exact formula for an average of L-series. Illionis J. Math. 1982, 26, 1-3. [CrossRef]

9. Zhang, W.P. On the mean values of Dedekind Sums. J. ThÉOrie Nombres Bordx. 1996, 8, 429-442. [CrossRef]

10. Zhao, J.H.; Wang, X. One kind hybrid character sums and their upper bound estimates. J. Inequalities Appl. 2018, 2018, 16. [CrossRef] [PubMed]

11. Pan, C.D.; Pan, C.B. Goldbach Conjecture; Science Press: Beijing, China, 2011.

12. Pan, C.D.; Pan, C.B. Basic Analytic Number Theory, (2e); Harbin Institute of Technology Press: Harbin, China, 2016.

13. Zacharias, R. Mollification of the fourth moment of Dirichlet L-functions. Acta Arith. 2019, 191, $201-257$. [CrossRef]

14. Xiu, Z.F.; Zhang, W.P. Some identity involving Dirichlet L-functions. Acta Arith. 2007, 130, 157-166. [CrossRef]

15. Liu, H.N.; Zhang, W.P. On the mean value of $L(m, \chi) L(n, \bar{\chi})$ at positive integers $m, n \geq 1$. Acta Arith. 2006, 122, 51-56. [CrossRef]

16. Wu, Z.X.; Zhang, W.P. On the mean value of $L(1, \chi)$. Bull. Korean Math. Soc. 2012, 49, 1303-1310. [CrossRef]

17. Zhang, W.P.; Han, D. On the Chebyshev polynomials and some of their reciprocal sums. Symmetry 2020, 12, 704. [CrossRef]

18. Ma, Y.K.; Lv, X.X. Some identities involving the reciprocal sums of one kind Chebyshev polynomials. Math. Probl. Eng. 2017, 2017, 4194579. [CrossRef]

(C) 2020 by the authors. Licensee MDPI, Basel, Switzerland. This article is an open access article distributed under the terms and conditions of the Creative Commons Attribution (CC BY) license (http:/ / creativecommons.org/licenses/by/4.0/). 